Nuclear Physics A470 (1987) 339-348

North-Holland, Amsterdam

\title{
THE MECHANISM OF THE ${ }^{7} \operatorname{Li}(d, 2 \alpha) n$ REACTION FROM $E_{d}=3$ TO $15 \mathrm{MeV}^{\star}$
}

\author{
R.E. WARNER, B.A. VAUGHAN ${ }^{1}$, J.A. DITUSA ${ }^{2}$, J.W. ROVINE and R.S. WAKELAND ${ }^{3}$ \\ Oberlin College, Oberlin, $\mathrm{OH} 44074$, USA \\ C.P. BROWNE, S.E. DARDEN and S. SEN ${ }^{4}$ \\ University of Notre Dame, Notre Dame, IN 46556, USA
}

A. NADASEN

University of Michigan, Dearborn, MI 48128, USA

A. BASAK ${ }^{5}$, T.R. DONOGHUE, T. RINCKEL and K. SALE

The Ohio State University, Columbus, $\mathrm{OH} 43210$, USA

G.C. BALL, W.G. DAVIES, A.J. FERGUSON and J.S. FORSTER

AECL Research Company, Chalk River Nuclear Laboratories, Chalk River, Ontario KOJ 1J0, Canada

Received 28 November 1986

(Revised 23 March 1987)

Abstract: The ${ }^{7} \mathrm{Li}(\mathrm{d}, 2 \alpha) \mathrm{n}$ reaction proceeds almost entirely through excitation and sequential decay of the 16.63 and $16.92 \mathrm{MeV}$ levels in ${ }^{8} \mathrm{Be}$, for incident energies 1 to $13 \mathrm{MeV}$ above their threshold. The energy dependence for forming these levels with the neutron emitted at $0^{\circ}$ is approximately that predicted assuming the neutron is a spectator from the incident deuteron. None of the individual spectra, the angular dependence of the cross section at fixed $E_{\mathrm{d}}$, or the bombarding-energydependence of the cross section for forming the levels is consistent with the involvement of a spectator neutron from the ${ }^{7} \mathrm{Li}$ target.

E

NUCLEAR REACTIONS ${ }^{7} \mathrm{Li}(\mathrm{d}, 2 \alpha), \quad E=3-15 \mathrm{MeV}$; measured $\sigma\left(\theta_{1}, \theta_{2}\right)$ vs energy difference. ${ }^{8} \mathrm{Be}$ deduced levels. Enriched ${ }^{7} \mathrm{LiF}$ targets. Deduced reaction mechanism.

\section{Introduction}

The ${ }^{7} \mathrm{Li}+\mathrm{d}$ reactions have long been of interest, partly because $\alpha$-particles are so copiously produced through the formation and decay of the intermediate nuclei

* Research supported by the National Science Foundation under Grants PHY 85-21042, PHY 84-21302, PHY 82-03699, and PHY 83-04886.

1 Present address: Physics Department, Stanford University, Stanford, CA 94305.

2 Present address: Physics Department, Cornell University, Ithaca, NY 14853.

${ }^{3}$ Present address: Physics Department, Indiana University, Bloomington, IN 47405.

4 Present address: Los Alamos National Laboratory, Los Alamos, NM 87545.

5 Present address: Rajshahi University, Rajshahi, Bangladesh. 
${ }^{5} \mathrm{He}$ and ${ }^{8} \mathrm{Be}$, in both their ground and excited states. One interesting question is whether these states are fed by stripping or compound nucleus formation. Measurements ${ }^{1,2}$ ) of inclusive $\alpha$-particle spectra at low bombarding energies show resonances corresponding to known states in the compound nucleus ${ }^{9} \mathrm{Be}$.

More recently, a coincidence measurement of the ${ }^{7} \mathrm{Li}(\mathrm{d}, 2 \alpha) \mathrm{n}$ reaction was performed by Lattuada et al. ${ }^{3}$ ) at $E_{\mathrm{d}}=2 \mathrm{MeV}$. At this energy the mixed-isospin ${ }^{8} \mathrm{Be}(16.63 \mathrm{MeV})$ state can be formed, though below its centroid; however, most of their measurements were made with sufficiently small included angles between detectors that they observed primarily the sequential decay (SD) of the ${ }^{5} \mathrm{He}$ ground state. Moreover, the yield of this state decreased less rapidly than expected as the (equal) $\alpha$-detection angles were decreased from $74^{\circ}$ to $66^{\circ}$. This yield was decomposed into two components: that from $\mathrm{SD}$ of ${ }^{5} \mathrm{He}$, and a direct reaction component resulting from a quasifree interaction ${ }^{6} \mathrm{Li}(\mathrm{d}, 2 \alpha)$ between the projectile and a ${ }^{6} \mathrm{Li}$ cluster in the target. The latter component was analyzed using the plane wave impulse approximation (PWIA), and a momentum distribution for a $\mathrm{p}_{3 / 2}$ neutron in ${ }^{7} \mathrm{Li}$ was deduced.

In a similar experiment, Kasagi et al. $\left.{ }^{4}\right)$ interpreted their ${ }^{9} \mathrm{Be}\left({ }^{3} \mathrm{He}, 2 \alpha\right) \alpha$ measurements at $4 \mathrm{MeV}$ as evidence for a quasifree reaction between the projectile and a ${ }^{5} \mathrm{He}$ cluster in the target. They deduced this cluster's momentum distribution from the $\alpha+\alpha$ coincidence energy spectrum, again using the PWIA.

It is surprising that reactions should be quasifree at such low bombarding energies as those employed in refs. ${ }^{3,4}$ ), where the projectile wavelength greatly exceeds nuclear dimensions. Another counterintuitive aspect of the Lattuada interpretation ${ }^{3}$ ) is that, if there is to be a spectator neutron in the ${ }^{7} \mathrm{Li}(\mathrm{d}, 2 \alpha) \mathrm{n}$ reaction, the one in the deuteron seems a more likely candidate since it is so much less tightly bound. Finally, even if these reactions are found to be quasifree, a more sophisticated theoretical framework than the PWIA is needed to extract the cluster momentum distributions. Distortion effects are certainly large and must be taken into account, along with the interference between the various reaction channels. It would then be useful to test whether the neutron momentum distributions in ${ }^{7} \mathrm{Li}$, as measured by the $(\mathrm{d}, 2 \alpha)$ and various knockout reactions, are in agreement.

The simpler questions of whether the ${ }^{7} \operatorname{Li}(\mathrm{d}, 2 \alpha) \mathrm{n}$ reaction is quasifree and, if so, in which nucleus the spectator neutron originates, may be answered more definitively by data taken over a range of bombarding energies than at a single energy. The different internal velocity distributions of the $s_{1 / 2}$ neutron in ${ }^{2} \mathrm{H}$ and the $\mathrm{p}_{3 / 2}$ neutron in ${ }^{7} \mathrm{Li}$ result in quite different predictions for the energy dependence of the reaction yield. Therefore, to test whether the reaction mechanism deduced ${ }^{3}$ ) at $E_{\mathrm{d}}=2 \mathrm{MeV}$ continues to operate at higher energies, we have made ${ }^{7} \mathrm{Li}(\mathrm{d}, 2 \alpha) \mathrm{n}$ coincidence measurements in the 3 to $15 \mathrm{MeV}$ range. In this region, excitation of the mixed-isospin doublet levels $\left.{ }^{5}\right)$ in ${ }^{8} \mathrm{Be}(16.63$ and $16.92 \mathrm{MeV})$ bccomes possible over a large region of phase space. Because of the strong single-particle character ${ }^{6}$ ) of these levels which lie just below the $p+{ }^{7} \mathrm{Li}$ threshold, one expects that their sequential decay 
would make a large contribution to the reaction yield; in earlier unreported measurements, we found that it dominates all others. At these bombarding energies there exist two equal-angle pairs at which $\alpha$-particles from SD of either level may be detected in coincidence. In these two cases, the undetected neutron is emitted at either $0^{\circ}$ or $180^{\circ}$ in the overall c.m. system, while in both cases the $\alpha$-particles emerge at $90^{\circ}$ in the ${ }^{8} \mathrm{Be}$ rest frame. Emission of the neutron at $0^{\circ}$ is analogous to forward stripping and might result from deuteron breakup. Likewise, if neutrons observed at $180^{\circ}$ resulted from a quasi-elastic reaction between the projectile and a ${ }^{6} \mathrm{Li}$ cluster in ${ }^{7} \mathrm{Li}$, they would be expected to display the momentum distribution of a $\mathrm{p}_{3 / 2}$ nucleon from ${ }^{7} \mathrm{Li}$. In fact, both the shapes of our measured spectra and the dependence of yields upon bombarding energy differ significantly from those predicted by either the ${ }^{7} \mathrm{Li}$ or ${ }^{2} \mathrm{H}$ spectator model.

\section{Experimental procedure}

Initial studies of this reaction were performed at the Chalk River and Ohio State University van de Graaff accelerators. Final data were taken at the Notre Dame University tandem van de Graaff, using 3 to $15 \mathrm{MeV}$ deuteron beams (typically $75 \mathrm{nA}$ ) incident upon a ${ }^{7} \mathrm{LiF}$ target. Coincident reaction products were detected in $2 \mathrm{~mm}$ thick Si detectors placed coplanar with and on opposite sides of the beam; their rectangular defining apertures, which subtended 2.64 and $2.81 \mathrm{msr}$, had heights of about three times their widths. Measurements of the ${ }^{7} \mathrm{Li}(\mathrm{p}, 2 \alpha)$ reaction provided energy calibration. The coincidence yield from this reaction as a function of the included angle between detectors showed that the angle markings in the scattering chamber were accurate to about $0.1^{\circ}$.

Signals were processed by conventional fast-slow electronics. Digitized signals from the two detectors and a time-to-digital converter were stored in event mode on magnetic tape. Random coincidence rates were found, during analysis, to be typically $3 \%$ of the total coincidence rates. The dead time was found to be less than $1 \%$ by scaling the current integrator pulses both directly and after passage through the event handler.

The target thickness was determined to be $0.94 \pm 0.05 \mathrm{mg} / \mathrm{cm}^{2}$ with a ${ }^{212} \mathrm{Bi}$ source; the 6.050 and $8.785 \mathrm{MeV} \alpha$-particle groups from this source lost 0.61 and $0.42 \mathrm{MeV}$ in the target, respectively. Isotopic purity was established by searching for ${ }^{6} \mathrm{Li}(\mathrm{d}, 2 \alpha)$ events at $E_{\mathrm{d}}=15 \mathrm{MeV}$ with the detectors at the appropriate symmetric geometry $\left(\theta_{1}=\theta_{2}=67.7^{\circ}\right)$ for this reaction; negligible coincidence yield was observed.

The detectors were large enough to detect $\alpha+\alpha$ coincidences from sequential decay of both the ${ }^{8} \mathrm{Be}(16.63,16.92)$ levels, at both of the equal-angle pairs previously described. For bombarding energies above $10 \mathrm{MeV}$ the forward detector angles (corresponding to neutron emission at $180^{\circ} \mathrm{c} . \mathrm{m}$.) were so near the symmetric angle pairs for $d+{ }^{7} \mathrm{Li}$ and $d+{ }^{19} \mathrm{~F}$ elastic scattering coincidences $\left(55.5^{\circ}\right.$ and $58.3^{\circ}$, respectively) that data rates would have been prohibitive. Therefore, measurements were 
made at both angle pairs for bombarding energies from 3 to $10 \mathrm{MeV}$, at $1 \mathrm{MeV}$ intervals; for $E>10 \mathrm{MeV}$, measurements were made only for $0^{\circ}$ neutron emission.

It was kinematically possible for contaminant events from the ${ }^{19} \mathrm{~F}(\mathrm{~d}, 2 \alpha){ }^{13} \mathrm{C}$ reaction, with $Q=+3.675 \mathrm{MeV}$, to underly the group being studied in a few cases (neutron at $0^{\circ}, E_{\mathrm{d}} \geqslant 12 \mathrm{MeV}$ ). In these cases, the neutron from ${ }^{7} \mathrm{Li}(\mathrm{d}, 2 \alpha)$ n was so energetic that, despite the $Q$-value of $+15.122 \mathrm{MeV}$, the combined energies of the $\alpha$-particles from the two reactions were nearly equal. Examination of twodimensional energy spectra at lower energies where the ${ }^{19} \mathrm{~F}(\mathrm{~d}, 2 \alpha)$ group would have been well resolved showed no discernible yield from this (presumably, direct) reaction. Moreover, data were taken at $E_{\mathrm{d}}=15 \mathrm{MeV}$ at an intermediate symmetric geometry (detectors at $73^{\circ}$ ) where the groups from the two reactions would have been well separated. Since no yield from ${ }^{19} \mathrm{~F}(\mathrm{~d}, 2 \alpha)$ was observed here either, we assumed that contamination from this reaction was negligible. No other three body final states accessible from $\mathrm{d}+{ }^{19} \mathrm{~F}$ can be confused with the reaction being studied since their $Q$-values are too negative.

We determined that the contamination of our spectra by $\alpha$-particles which follow the $\beta$-decay of ${ }^{8} \mathrm{Li}$ is negligible. Some ${ }^{8} \mathrm{Li}$ nuclei will be produced in the target, particularly at low bombarding energies. However, conservation of energy between the two leptons and two $\alpha$-particles precludes $\alpha+\alpha$ coincidence detection with $E_{\alpha} \geqslant 0.3 \mathrm{MeV}$ at the included angles utilized in this experiment. Any e $+\alpha$ coincidences would be spread out over the $E_{1}-E_{2}$ plane rather than confined to a three-body kinematic locus. The density of events outside the loci of the reaction being studied was sufficiently low to show that no correction for this effect was needed.

\section{Results and discussion}

Observed cross sections for the ${ }^{7} \mathrm{Li}(\mathrm{d}, 2 \alpha) \mathrm{n}$ reaction, for several optimum equalangle pairs for detecting $\alpha+\alpha$ coincidences from sequential decay (SD) of the ${ }^{8} \mathrm{Be}(16.63,16.92)$ states, are presented in fig. 1 . The error bars include only statistical uncertainties. These coincidence cross sections are plotted vs. the energy difference of the detected particles, to exploit the symmetry of the detection geometry. In general the SD process dominates the reaction yield; generally, the channels outside the peak have no more than $2 \%$ of the counts at the center of the resonance. The yield was so large, and the spectra were so clean, that small corrections for amplifier gain drifts were made by making the centroids of the SD peaks correspond to the known equal- $\alpha$-particle energies.

The yield for a given energy difference was assumed proportional to the solid angle $\mathrm{d} \Omega_{1}$ of one detector, the neutron emission solid angle $\mathrm{d} \Omega_{\mathrm{n}}$ permitted by detection of the second $\alpha$-particle, the three-body phase-space factor $\rho_{3}$, and a nuclear reaction probability $f\left(\Omega_{1}, \Omega_{n}, E_{1}-E_{9}\right)$ appropriate to one of the three models which were tested. Thus, predictions for the peak shapes were made by integrating

$$
Y\left(E_{1}-E_{2}\right)=\int \rho_{3} f\left(\Omega_{1}, \Omega_{n}, E_{1}-E_{2}\right) \mathrm{d} \Omega_{1} \mathrm{~d} \Omega_{n} \mathrm{~d} E_{1}
$$



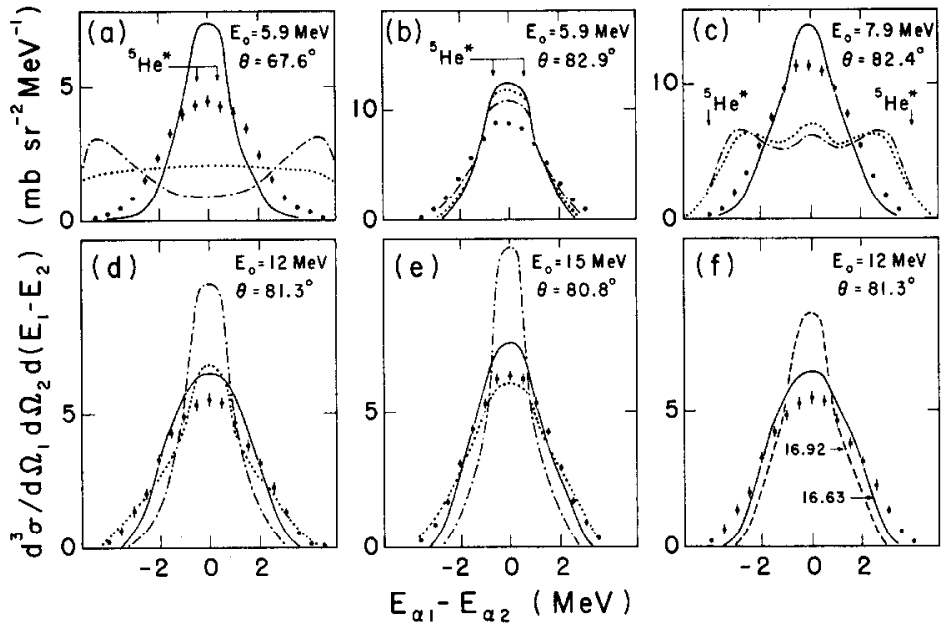

Fig. 1. Coincidence cross sections for the ${ }^{7} \mathrm{Li}(\mathrm{d}, 2 \alpha) \mathrm{n}$ reaction at indicated bombarding energies and equal coplanar detection angles. Predictions in 1a through 1e are for sequential decay (solid lines) of ${ }^{8} \mathrm{Be}(16.63 \mathrm{MeV})$ and for a spectator neutron originating in ${ }^{2} \mathrm{H}$ (dotted lines) or ${ }^{7} \mathrm{Li}$ (dot-dash lines). In fig. 1f, predicted line shapes for ${ }^{8} \mathrm{Be}(16.63$ and $16.92 \mathrm{MeV})$ are compared; the dashed line is the prediction for the $16.92 \mathrm{MeV}$ level. Arrows mark centroids for possible sequential decay of the ${ }^{5} \mathrm{He}$ first excited state.

over the solid angles of both detectors and all detected particle energies $E_{1}$ consistent with one bin-width of energy difference. For the sequential decay model, the reaction probability $f$ was a Breit-Wigner expression whose denominator contained the relative energy of the two detected $\alpha$-particles and the excitations and widths of these ${ }^{8} \mathrm{Be}$ states as given in ref. ${ }^{5}$ ). For the model in which a spectator neutron originated in ${ }^{7} \mathrm{Li}, f$ was taken to be a gaussian function in the undetected neutron's laboratory momentum; the two parameters of this function (centroid $=79 \mathrm{MeV} / c$, $\mathrm{FWHM}=34 \mathrm{MeV} / \mathrm{c}$ ) were those found in ref. ${ }^{3}$ ) to describe the momentum distribution of neutrons in ${ }^{7} \mathrm{Li}$. Finally, for testing the possibility that a spectator neutron came from the deuteron, $f$ was taken to be the nucleon momentum distribution derived from a Hulthen wavefunction ${ }^{7}$ ); the argument of this distribution is the neutron momentum relative to the projectile c.m. Thus, both spectator models were equivalent to plane wave treatments. All predictions were renormalized to reproduce the total measured cross section within an energy difference interval $\left|E_{1}-E_{2}\right| \leqslant$ 4.2 MeV.

The predictions for sequential decay of the $16.63 \mathrm{MeV}$ level in ${ }^{8} \mathrm{Be}$ and for a spectator neutron originating in ${ }^{2} \mathrm{H}$ or ${ }^{7} \mathrm{Li}$ are compared with experiment in fig. 1 . The effects of instrumental broadening have been included in these calculations. In general the results for sequential decay give nearly the correct spectral shapes but do not always reproduce the observed width. The predictions for spectator neutrons are in disagreement with experiment at low bombarding energies, but give reasonably 
good fits at $E_{d} \geqslant 10 \mathrm{MeV}$ for geometries corresponding to a spectator neutron from ${ }^{2} \mathrm{H}$ at $0^{\circ}$.

In fig. If predictions for the sequential decay of the 16.63 and $16.92 \mathrm{MeV}$ levels are compared. Usually the $16.92 \mathrm{MeV}$ level gives a more narrow, and consequently less acceptable, fit. The lower and upper levels are considered ${ }^{6}$ ) to have $p+{ }^{7} \mathrm{Li}$ and $\mathrm{n}+{ }^{7} \mathrm{Be}$ single-particle characters, respectively; moreover, only the lower level participates $\left.{ }^{8}\right)$ in the direct ${ }^{7} \mathrm{Li}(\mathrm{p}, \gamma)$ capture reaction. Therefore it is not surprising that the data are better fitted by assuming SD through the lower level, but the possibility of contributions from both levels cannot be excluded.

Cross sections measured at lower energies ${ }^{1-3}$ ) show the influence of sequential decay of the ground and first excited states of ${ }^{5} \mathrm{He}$. Observation of the ground state is excluded by our experimental conditions. However, the first excited state can be formed at its centroid by one $\alpha$-particle and the undetected neutron at locations marked in some of the figures, and the tails of this broad state would extend through regions of all of our spectra. Evidence that it contributes little to the observed yield will be presented later.

As a further test of both spectator models our observed cross sections, integrated over an energy difference interval of $4.2 \mathrm{MeV}$, are plotted vs. bombarding energy in fig. 2. The model energy dependences obtained from eq. (1) have been arbitrarily and separately normalized to the data for $\phi_{\mathrm{n}}=0^{\circ}$ and $180^{\circ}$. For neutrons emitted

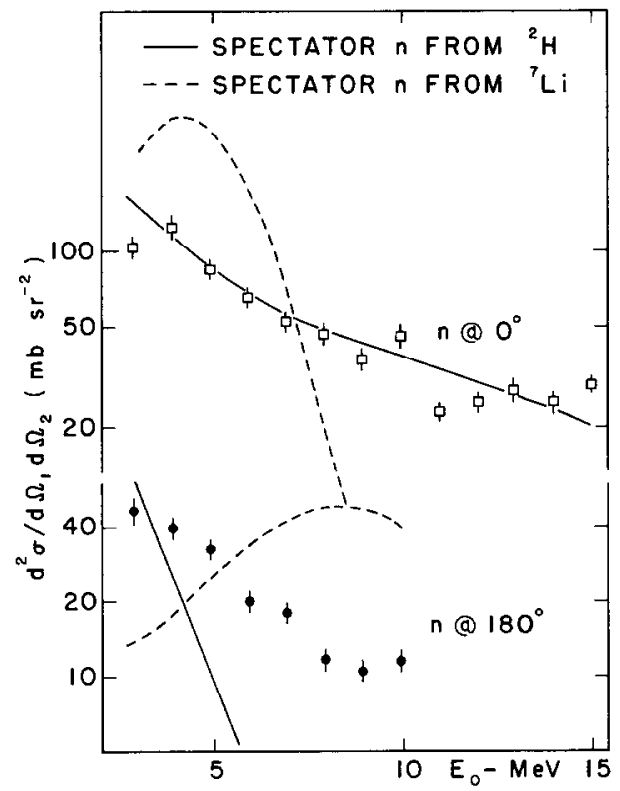

Fig. 2. Cross sections for ${ }^{7} \mathrm{Li}(\mathrm{d}, 2 \alpha)$ n, with $\mathrm{n}$ emitted at $0^{\circ}$ and $180^{\circ} \mathrm{c}$.m., integrated over the interval $\left|E_{\alpha 1}-E_{\alpha 2}\right| \leqslant 4.2 \mathrm{MeV}$. Predictions are for a spectator neutron originating in ${ }^{2} \mathrm{H}$ (solid lines) or ${ }^{7} \mathrm{Li}$ (dashed lines). Detection angles are given in table 1. 
near $0^{\circ}$ (upper part of fig. 2) the energy dependence is roughly reproduced by the neutron-from- ${ }^{2} \mathrm{H}$ spectator model. Thus the forward stripping yield is conditioned by the probability of finding a neutron with the correct final momentum in the deuteron. However, fig. 1 shows that the line shape of the ${ }^{8} \mathrm{Be}$ state, not the nucleon momentum distribution, determines the spectrum at low bombarding energies. Presumably, the most favorable conditions for observing spectator neutrons from ${ }^{7} \mathrm{Li}$ are at $180^{\circ} \mathrm{c}$.m.; however, the fit for this case is worst of all. Thus both spectator models fail to fit the data at $180^{\circ}$.

The dimensionless ratio of the measured cross sections for forward and backward neutron emission is plotted in fig. 3 for the bombarding energy range over which both were measurable. Absolute predictions of this ratio were made using eq. (1); both measured and predicted ratios are for an energy difference interval of $4.2 \mathrm{MeV}$. Both spectator model predictions (solid lines) of the energy dependence differ strongly from the data. Thus, at best, each model can work only in restricted regions of phase space. For comparison, we show also a prediction (dashed line) obtained by assuming SD of the ${ }^{8} \mathrm{Be}(16.63 \mathrm{MeV})$ level, with equal probabilities for forward and backward neutron emission. This assumption is clearly oversimplified since it excludes all nuclear structure information; however, it is interesting to note that the prediction has nearly as weak an energy dependence as the experimental data. In general one would expect the measured ratio to be larger since stripping cross sections at $0^{\circ}$ generally exceed those at $180^{\circ}$.

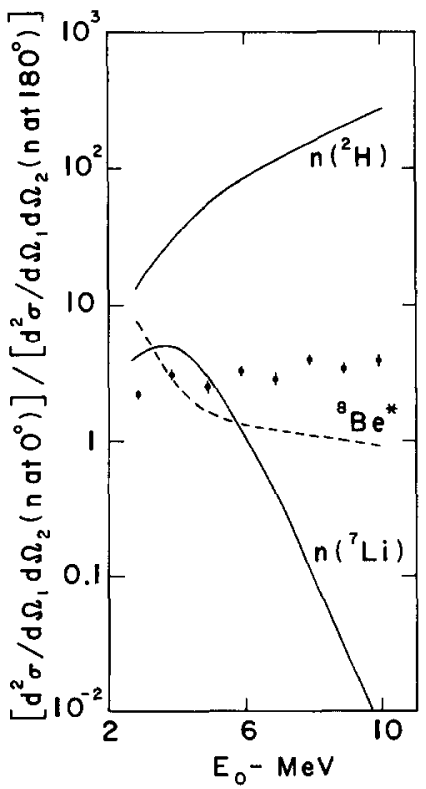

Fig. 3. Ratios of cross sections (from fig. 2) for forward and backward neutron emission in the ${ }^{7} \mathrm{Li}(\mathrm{d}, 2 \alpha) \mathrm{n}$ reaction. Predictions, not renormalized, are for the two spectator models and sequential decay of ${ }^{8} \mathrm{Be}(16.63)$. 
To explore the possibility that other levels in ${ }^{8} \mathrm{Be}$ and/or ${ }^{5} \mathrm{He}$ might be excited by the reaction, measurements were made at $E_{\mathrm{d}}=10 \mathrm{MeV}$ for several equal-angle pairs including those for the $16.63 \mathrm{MeV}$ level. Fig. 4 displays the cross sections, for an energy difference interval of $1.3 \mathrm{MeV}$, plotted vs. both detection angle and excitation in the ${ }^{5} \mathrm{He}$ and ${ }^{8} \mathrm{Be}$ system. The cross sections drop precipitously from the two main peaks. A smaller maximum appears at about $68^{\circ}$, near where one expects SD from the three known $\alpha$-emitting states near $20 \mathrm{MeV}$ excitation in ${ }^{8} \mathrm{Be}$. SD of the very broad (FWHM $=4 \pm 1 \mathrm{MeV}$ ) ${ }^{5} \mathrm{He}$ first excited state could occur in this region but would yield a broader peak than is observed. Both spectator models (dashed and dot-dashed lines) predict very small cross sections at the intermediate angles.

The bombarding energies and detection angles employed in this study, and the measured cross sections for a $4.2 \mathrm{MeV}$ energy difference interval, are summarized in table 1. Statistical uncertainties for this large interval are always less than $2 \%$, and uncertainties shown in both this table and figs. 2 through 4 are based primarily upon the $10 \%$ target thickness uncertainty.

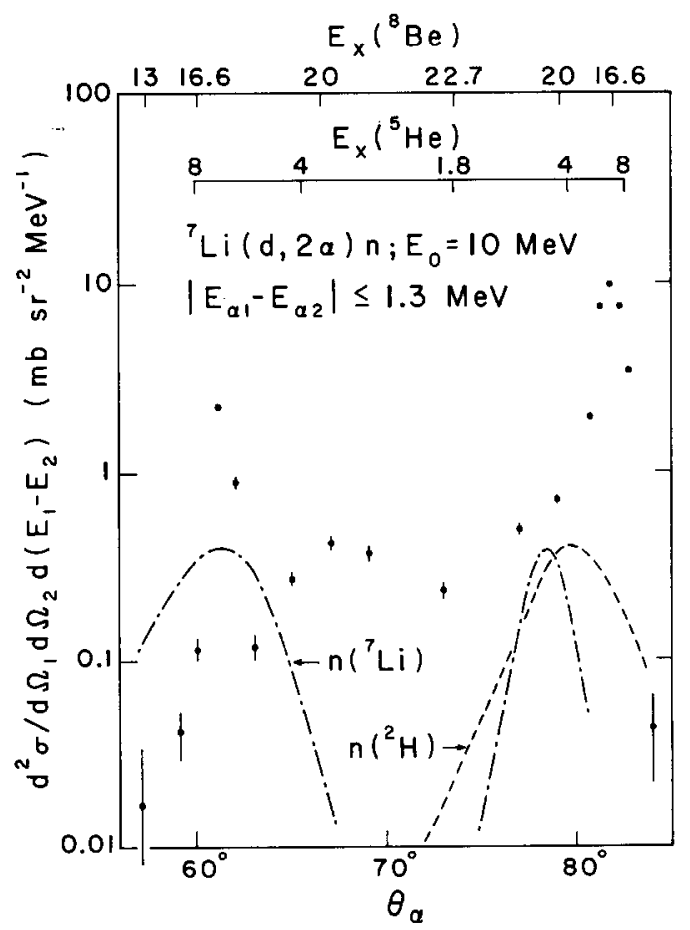

Fig. 4. Measured cross sections at $E_{\mathrm{d}}=10 \mathrm{MeV}$ for ${ }^{7} \mathrm{Li}(\mathrm{d}, 2 \alpha)$ n, integrated over the interval $\left|E_{\alpha 1}-E_{\alpha 2}\right| \leqslant$ 1.3 MeV. Data and predictions for both spectator models are plotted versus detection angles and excitations in the ${ }^{5} \mathrm{He}$ and ${ }^{8} \mathrm{Be}$ systems. 
TABLE 1

Cross sections for ${ }^{7} \mathrm{Li}(\mathrm{d}, 2 \alpha) \mathrm{n} ;\left|E_{\alpha 1}-E_{\alpha 2}\right| \leqslant 4.2 \mathrm{MeV}$

\begin{tabular}{rccccc}
\hline & \multicolumn{2}{c}{ Neutron at $0^{\circ}$ c.m. } & & \multicolumn{2}{c}{ Neutron at $180^{\circ}$ c.m. } \\
\cline { 2 - 3 } \cline { 5 - 6 }$(\mathrm{MeV})$ & $\begin{array}{c}\theta_{\alpha, \mathrm{lab}} \\
(\text { deg. })\end{array}$ & $\begin{array}{c}\mathrm{d}^{2} \sigma / \mathrm{d} \Omega_{1} \mathrm{~d} \Omega_{2} \\
\left(\mathrm{mb} / \mathrm{sr}^{2}\right)\end{array}$ & $\begin{array}{c}\theta_{\alpha, \text { lab }} \\
(\text { deg. })\end{array}$ & $\begin{array}{c}\mathrm{d}^{2} \sigma / \mathrm{d} \Omega_{1} \mathrm{~d} \Omega_{2} \\
\left(\mathrm{mb} / \mathrm{sr}^{2}\right)\end{array}$ \\
\hline 2.85 & 83.3 & $104 \pm 11$ & 75.2 & $47 \pm 4.9$ \\
3.88 & 83.2 & $126 \pm 13$ & 72.2 & $40 \pm 4.2$ \\
4.90 & 83.1 & $85 \pm 9$ & 69.7 & $33 \pm 3.5$ \\
5.91 & 82.9 & $66 \pm 7$ & 67.6 & $20 \pm 2.3$ \\
6.92 & 82.6 & $53 \pm 6$ & 65.7 & $18 \pm 2.1$ \\
7.93 & 82.4 & $47 \pm 5$ & 64.0 & $11.7 \pm 1.3$ \\
8.94 & 82.1 & $37 \pm 4$ & 62.5 & $10.6 \pm 1.3$ \\
9.97 & 81.8 & $46 \pm 5$ & 61.1 & $11.5 \pm 1.3$ \\
10.94 & 81.6 & $23 \pm 2.5$ & & \\
11.95 & 81.3 & $25 \pm 2.7$ & & \\
12.97 & 81.1 & $28 \pm 3.0$ & & \\
13.97 & 80.9 & $25 \pm 2.7$ & & \\
14.97 & 80.8 & $29 \pm 3.1$ & & \\
\hline
\end{tabular}

\section{Conclusions}

Most of the yield of the ${ }^{7} \mathrm{Li}(\mathrm{d}, 2 \alpha) \mathrm{n}$ reaction in the region of phase space which we investigated results from sequential decay of excited ${ }^{8} \mathrm{Be}$ levels, mainly the mixed-isospin levels at 16.63 and $16.92 \mathrm{MeV}$. Individual spectra are fitted most consistently by assuming SD of these two levels. There is no evidence of the participation of the first excited state of ${ }^{5} \mathrm{He}$.

When the data are compared with the predictions of two plane wave spectator models, the discrepancies appear to be too great to be attributed to possible distortion effects. We therefore conclude that spectator processes are not dominant for this reaction at these bombarding energies. In particular neither the spectral shapes, nor the angular or energy dependence of the cross section, are consistent with the involvement of a spectator neutron from ${ }^{7} \mathrm{Li}$. However, the bombarding-energy dependence of the cross section at those geometries where the neutron is emitted at $0^{\circ}$ is approximately that expected when a spectator neutron originates in the incident deuteron.

It would be worthwhile to use this reaction at still higher bombarding energies to search for spectator neutrons from both ${ }^{2} \mathrm{H}$ and ${ }^{7} \mathrm{Li}$. It would then be interesting to compare the momentum distribution of neutrons from ${ }^{7} \mathrm{Li}$ with that obtained in, e.g., a (p, pn) knockout experiment. This distribution could be reliably extracted only by comparing the data with a full calculation which took account of both distortion effects and interference between the dominant reaction channels. 
We wish to thank E.D. Berners, J. Gaiser, and J. Kaiser for their extensive help with the experimental equipment, and R. Vojtech and $J$. Hinnefield for their generous assistance in recovering the data from the magnetic tapes.

\section{References}

1) P. Paul and D. Kohler, Phys. Rev. 129 (1963) 2698

2) E. Friedland and I. Venter, Z. Phys. 243 (1971) 126

3) M. Lattuada et al., Nuov. Cim. 72A (1982) 51

4) J. Kasagi et al., Nucl. Phys. A239 (1975) 233

5) F. Ajzenberg-Selove, Nucl. Phys. A320 (1979) 1

6) J.B. Marion, Phys. Lett. 14 (1965) 315

7) M.J. Moravcsik, Nucl. Phys. 7 (1958) 113

8) J.B. Marion and M. Wilson, Phys. Lett. 14 (1965) 313 